\title{
Study on chloride ion diffusion characteristics of reactive powder concrete under different loads
}

\author{
Yan Xin-e $e^{1, *}$ \\ ${ }^{1}$ School of civil engineering, Xi'an traffic engineering institute, Xi'an, Shaanxi, China
}

\begin{abstract}
Reactive powder concrete (RPC) is a new cement-based material with ultra-high strength, high durability, high toughness and good volume stability. The study of chloride corrosion behavior is of great significance to the application of RPC in complex service environment. In this paper, the chloride ion immersion experiment is carried out to investigate the change rule of chloride ion concentration on the surface of RPC with soaking time under different loads. The results show that with the extending of soaking time, the chloride ion concentration of RPC surface gradually increases and tends to be stable, and the different depths from RPC surface also show similar rules. The results also show that the chloride ion diffusion characteristics of RPC under different loads are different, and the chloride ion diffusion rate under bending stress is higher than that under compressive stress.
\end{abstract}

\section{Introduction}

With the development of society and economy, people's requirements for building facilities are increasing, especially with more and more high-rise, large-span, lightweight structure and harsher environment appearing in modern buildings, especially requirements for the mechanical properties and durability of concrete materials. Under such a circumstance, the reactive powder concrete (RPC) provides a new way to solve these problems. ${ }^{[1-2]}$

RPC is a new type of cement-based material with ultra-high strength, high durability, high toughness, and good volume stability developed by the French company Bouygues in the middle of the last century following with high-strength and high-performance concrete ${ }^{[3]}$. Compared with other high-performance concretes, the important feature of RPC is that it removes coarse aggregates according to the principle of compaction, which makes its matrix compact and has high compressive strength and durability. By adding steel fibers to improve the ductility of high-strength concrete materials, the flexural and tensile strength have been greatly improved, and good toughness has also been obtained ${ }^{[4]}$. In actual projects, using reactive powder concrete to replace ordinary concrete or high-strength concrete can not only reduce the cross-sectional size of the structure and the amount of reinforcement, improve the durability and safety of the structure, but also design the new structure type that cannot be designed by ordinary concrete and high-strength concrete. Especially in the harsh service environment, RPC shows more superior durability ${ }^{[5-6]}$.

However, chloride salt damage is still one of the most important factors affecting the durability of RPC structures ${ }^{[7-8]}$, and the chloride salt damage is not only related to the characteristics of the concrete structure itself, but also to the service environment ${ }^{[9]}$. This paper mainly investigated the chloride ion diffusion characteristics of RPC under different stress loads, and analyzed the time-varying law of chloride ion concentration on the surface of RPC and the law of chloride ion diffusion in RPC.

\section{Experiment}

\subsection{Materials}

The cement used in this study is ordinary Portland cement with $\mathrm{P}$. O 42.5, and its quality requirements meet the standards of GB175-2020 "General Portland Cement". The main chemical composition and physical and mechanical performance indicators are shown in Table 1 and Table 2. Silica fume is produced by a silica fume factory in Xi' an, China. The $\mathrm{SiO}_{2}$ content is greater than $95 \%$, and the specific surface area is greater than $152000 \mathrm{~m}^{2} / \mathrm{kg}$. Quartz sand is divided into coarse-grain sand, medium-grain sand and fine-grain sand. The coarse-grain sand is $1.25-0.63 \mathrm{~mm}$, medium-grain sand is $0.63-0.315 \mathrm{~mm}$, fine-grain sand is less than $0.315-0.16$ $\mathrm{mm}$, the distribution ratio of these three particle sizes is 1:2:1; the quartz powder particle size is less than $45 \mu \mathrm{m}$, and the $\mathrm{SiO}_{2}$ content is greater than $97 \%$; The length of the steel fiber is $12 \mathrm{~mm}$, the diameter is $0.35 \mathrm{~mm}$, and the addition amount of the steel fiber is $2 \%$ (volume fraction). Admixtures are defoamer and polycarboxylic acid superplasticizer, and the water reducing rate of the water reducing agent is $30 \%$. Based on the results of

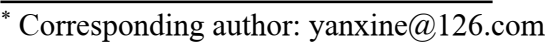


preliminary experimental research, the matching ratio of $\mathrm{RPC}$ is shown in Table $3^{[10]}$.

Table 1. The main components of P.O 42.5 cement

\begin{tabular}{|c|c|}
\hline Composition & Content(\%) \\
\hline $\mathrm{SiO}_{2}$ & 16.4 \\
\hline $\mathrm{Al}_{2} \mathrm{O}_{3}$ & 4.6 \\
\hline $\mathrm{CaO}$ & 61.8 \\
\hline $\mathrm{MgO}$ & 1.5 \\
\hline $\mathrm{SO}_{3}$ & 2.7 \\
\hline $\mathrm{Fe}_{2} \mathrm{O}_{3}$ & 4.2 \\
\hline L.O.I. & 2.8 \\
\hline
\end{tabular}

Table 2. Physical and mechanical properties of the cement

\begin{tabular}{|c|c|c|c|c|c|c|}
\hline \multirow{2}{*}{$\begin{array}{c}\text { Water } \\
\text { consumption } \\
/ \%\end{array}$} & \multicolumn{2}{|c|}{$\begin{array}{c}\text { Condensation } \\
\text { time / min }\end{array}$} & \multicolumn{2}{|c|}{$\begin{array}{c}\text { Flexural } \\
\text { strength / MPa }\end{array}$} & \multicolumn{2}{c|}{$\begin{array}{c}\text { Compressive } \\
\text { strength / } \\
\text { MPa }\end{array}$} \\
\cline { 2 - 7 } & Start & End & $3 \mathrm{~d}$ & $28 \mathrm{~d}$ & $3 \mathrm{~d}$ & $28 \mathrm{~d}$ \\
\hline 27 & 160 & 350 & 3.3 & 6.4 & 18.4 & 42.5 \\
\hline
\end{tabular}

Table 3. Mixing ratio for preparing reactive powder concrete

\begin{tabular}{|c|c|}
\hline Cement & 100 \\
\hline Quartz sand & 110 \\
\hline Quartz powder & 25 \\
\hline Silica fume & 20 \\
\hline Admixture & 3 \\
\hline Water-binder ratio & 0.2 \\
\hline
\end{tabular}

\subsection{Experimental scheme}

The preparation and curing of RPC refer to "Reactive Powder Concrete" (GB/T 31387-2015). The chloride ion diffusion experiment without stress and the chloride ion diffusion experiment under compressive stress use a cube test block of $100 \mathrm{~mm} \times 100 \mathrm{~mm} \times 100 \mathrm{~mm}$ under bending stress. The chloride ion diffusion experiment uses a prism test block of $100 \mathrm{~mm} \times 100 \mathrm{~mm} \times 400 \mathrm{~mm}$. Before the start of the experiment, all surfaces except the chloride ion diffusion study surface were sealed with epoxy resin.

In this paper, a $10 \% \mathrm{NaCl}$ solution is used as the medium, and the chloride salt immersion test under different continuous stress loads is tested. The experimental schematic diagram is shown in Fig. 1. The test samples soaked for $7 \mathrm{~d}, 14 \mathrm{~d}, 28 \mathrm{~d}, 60 \mathrm{~d}, 120 \mathrm{~d}, 180 \mathrm{~d}$ were taken out, and the powder at different depths of 0 $5 \mathrm{~mm}, 5-10 \mathrm{~mm}, 10-15 \mathrm{~mm}, 15-20 \mathrm{~mm}$ in the center of the test block was selected. The concentration of chloride ion in different surface depths of specimens was measured by potentiometric method to study the diffusion law of chloride ion in RPC, and the concentration of chloride ion on the surface of $0-5 \mathrm{~mm}$ was used to replace the concentration of chloride ion on the surface of RPC ${ }^{[11]}$. The compressive stress under the load environment is $50 \%$ of the ultimate compressive strength of RPC, and the bending stress is $50 \%$ of its ultimate flexural capacity. During the test, it is necessary to ensure that the concentration of the $\mathrm{NaCl}$ solution is stable at $10 \%$.

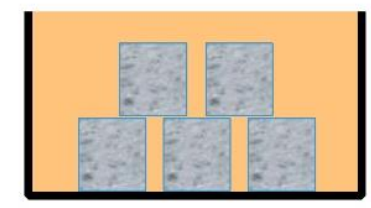

a
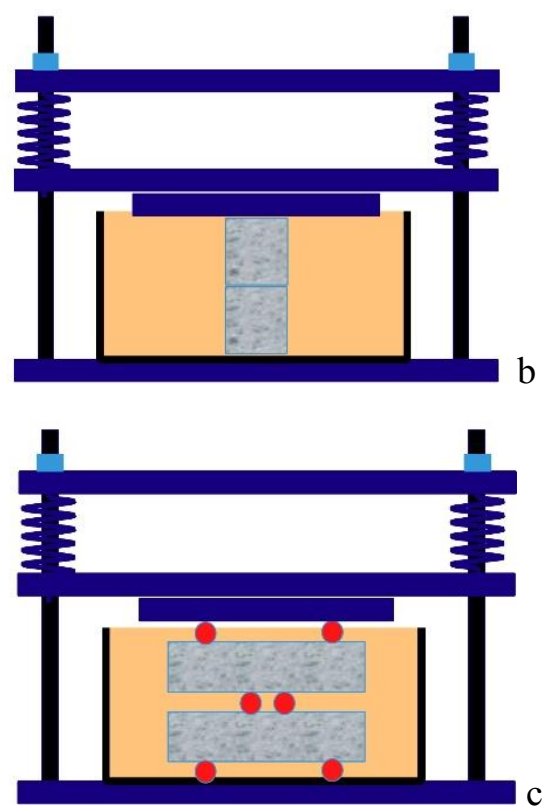

Fig. 1. Chloride ion diffusion experiment of RPC under different application forces (a: Chloride ion diffusion under stress-free; b: Chloride ion diffusion under compressive stress; c: Chloride ion diffusion under tensile stress)

\section{Results and discussion}

\subsection{Time-varying law of chloride ion concentration on the surface of RPC}

Researchers usually use Fick's second law to study the diffusion process of chloride ions in concrete, but the premise is that the chloride ion concentration on the concrete surface is constant. However, in actual situations, the chloride ion concentration on the concrete surface is not a constant value, but gradually accumulates over time to reach stability, which is a dynamic process. Fig. 2. shows the variation curve of RPC surface chloride ion concentration with immersion time under different stress loads. It can be seen from the figure that the surface chloride ion concentration of RPC under different stress loads increases with the extension of the immersion time, and its value first increases rapidly and then stabilizes; however, under the stressfree compressive stress environment, the initial increase 
speed of chloride ion concentration increases rapidly without the action of bending stress. Therefore, under different stress loads, the chloride ion concentration on the surface of RPC exhibits different laws over time.

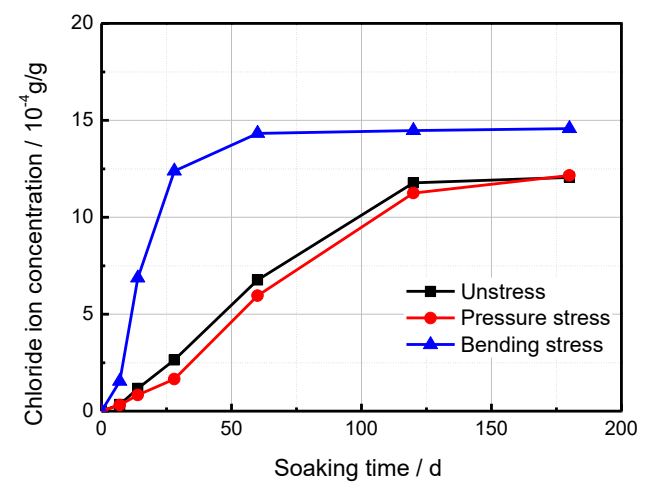

Fig. 2. Surface chloride ion concentration changes with immersion time

\subsection{The diffusion law of chloride ions under different stresses}

Fig. 3. shows the chloride ion diffusion characteristic curve in RPC over time under stress-free. It can be seen from the figure that as the immersion time increases, the diffusion depth of chloride ions is deeper, and the diffusion rate on the surface of the RPC is greater than the diffusion rate of chloride ions inside the RPC. This is mainly due to the fact that RPC is a high-density concrete with underdeveloped pore structure, which hinders the diffusion of chloride ions ${ }^{[12]}$. The surface diffusion rate of chloride ions in the initial stage of immersion in RPC is small, and then the diffusion rate increases, and finally the diffusion rate decreases as the concentration of chloride ions on the surface of RPC increases.

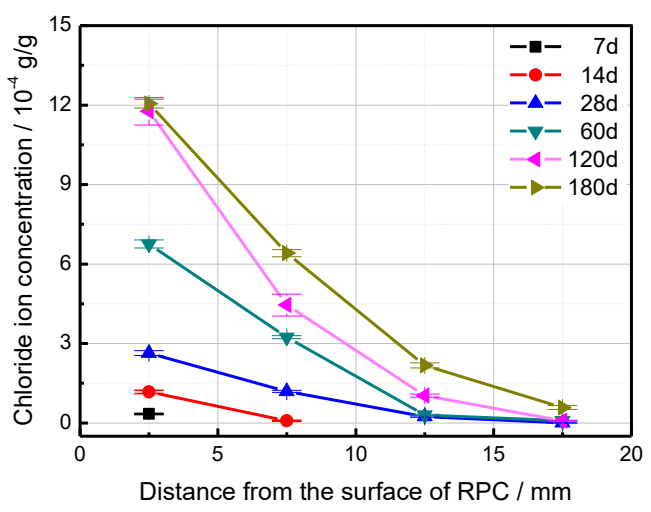

Fig. 3. Chloride ion diffusion characteristics of RPC during natural immersion

Fig. 4. shows the chloride ion diffusion characteristics of RPC under compressive stress load. Comparing the chloride ion diffusion characteristics of the RPC surface under the stress-free environment in Fig. 3 , the two have similar characteristics, indicating that the chloride ion diffusion characteristics of the RPC surface under compressive stress load have not changed significantly, but the chloride ion diffusion rate is slightly reduced. This is mainly because under compressive stress load, the RPC becomes denser and the pore structure is further reduced, and the diffusion resistance of chloride ions increases, resulting in a slight decrease in the diffusion rate of chloride ions.

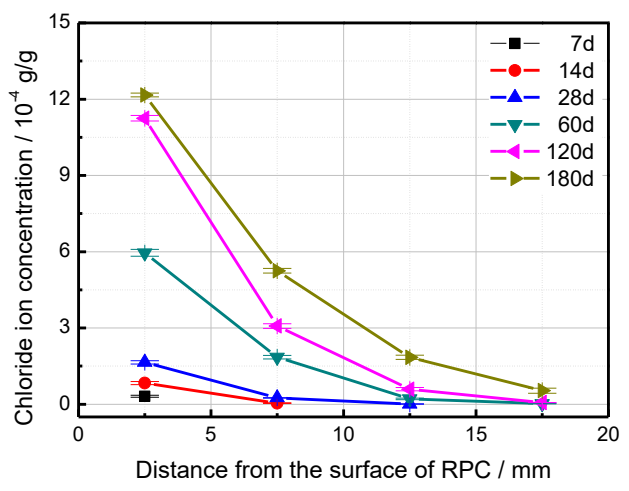

Fig. 4. Chloride ion diffusion characteristic curve of RPC under compressive stress load

Fig. 5. shows the chloride ion diffusion characteristics of RPC under bending stress load. Comparing the chloride ion diffusion characteristics of the RPC surface under stress-free and compressive stress loads, the chloride ion diffusion characteristics of the RPC surface under bending stress have changed significantly. It shows that the chloride ion concentration on the surface of RPC quickly reaches the equilibrium of diffusion, and the chloride ion concentration in the RPC is also significantly higher than the chloride ion concentration in the RPC under the action of stress-free and compressive stress, indicating that the chloride ion on the surface of the RPC diffuses under the action of bending stress. The characteristics have changed significantly. This is mainly because under the bending stress load, the pore structure of the surface and bulk of RPC has changed. Although the steel fiber contributes to the tensile strength of RPC, the change of RPC microscopic pore structure can cause the diffusion characteristics of chloride ions.

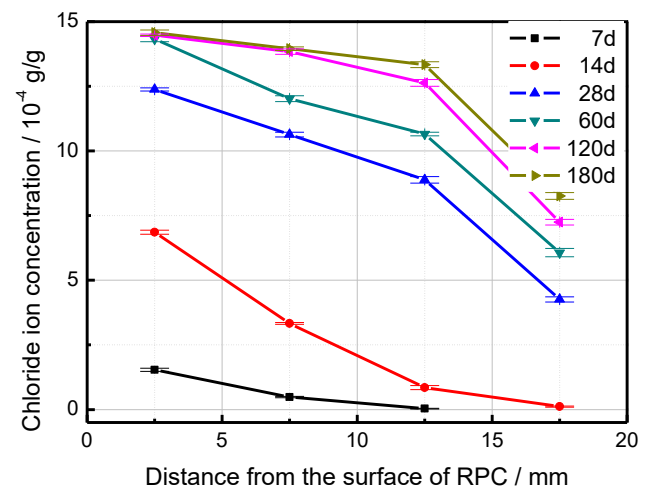

Fig. 5. Chloride ion diffusion characteristic curve of RPC under bending stress load

\subsection{Chloride ion diffusion model on RPC surface}

Comparing the chloride ion diffusion characteristic curves of RPC under different stresses, the results show 
that chloride ions are more likely to diffuse into the bulk phase of RPC under bending stress, which is consistent with the conclusion that the chloride ion concentration on the surface of RPC changes with time. However, the diffusion law of chloride ions on the surface of concrete can be described by linear equations, power function equations, exponential equations and logarithmic equations. Under standard conditions, the diffusion of chloride ions in concrete conforms to Fick's second law. The analytical formula for the diffusion of chloride ions in concrete under natural immersion is shown in equation (1).

$$
C(x, t)=C_{0}\left(1-\operatorname{erf}\left(\frac{x}{2 \sqrt{D t}}\right)\right)
$$

Where $C$ represents the chloride ion concentration at different depths, \%; $C_{0}$ represents the chloride ion concentration on the surface of the concrete, $\% ; x$ represents the depth from the concrete surface, $\mathrm{m} ; D$ is the diffusion coefficient of chloride ions, $\mathrm{m}^{2} / \mathrm{s}$; $\mathrm{t}$ is the duration of chloride ions diffusion, a; erf is the error function.

However, considering the comprehensive influence of the external environment of concrete, researchers need to modify Fick's second law. When the chloride ion diffusion coefficient is affected by the exposure time, its calculation needs to be corrected by introducing time parameters; when the concrete is under stress, the chloride ion diffusion becomes more complicated, so mathematical models need to be used for fitting. The fitting formula for the surface chloride ion concentration of RPC under bending stress is shown in equation (2), where the coefficient $r$ is the cumulative change law of the diffusion rate of reactive chloride ions, which is related to the external environmental factors where RPC is located.

$$
C(x, t)=C_{s o}-C_{s \max }\left(1-e^{r t}\right)
$$

The surface chloride ion concentration of RPC under stress-free and compressive stress is fitted by a mathematical model, and the fitting formula obtained is shown in equation (3).

$$
C(x, t)=A t^{4}+B t^{3}+C t^{2}+D t
$$

From the fitting formula of RPC's chloride ion surface concentration under different loads, it is more reasonable to use a power function to fit under bending stress, while it is more reasonable to use a polynomial to fit under compressive stress. However, the physical meaning of each parameter is not very clear in these mathematical models. Therefore, the mathematical equation description of the diffusion law of chloride ions in RPC under different stresses still needs more experimental data to verify, which is also the focus of subsequent research work.

\section{Conclusion}

In this paper, the $\mathrm{NaCl}$ solution immersion experiment is used to study the surface chloride ion concentration diffusion characteristics of RPC under different stresses. The results show that the chloride ion concentration on the surface of RPC changes little at the beginning of the experiment, and then gradually increases, and finally the chloride ion concentration on the surface tends to be stable; the compressive stress load has no significant effect on the diffusion of chloride ions in the RPC, while the diffusion rate of chloride ions in RPC increases significantly under bending stress, so there are significant differences in the diffusion law of chloride ions in RPC under different stress loads. Through the analysis of the mathematical model of the chloride ion concentration on the surface of RPC, it is found that under the action of bending stress, the fitting of the chloride ion concentration on the surface of RPC with a power exponential equation is more reasonable, while under the action of compressive stress, it is more reasonable to use polynomial fitting. All these conclusions will guide the prediction of chloride ion corrosion behavior of RPC, so as to put forward effective protection measures.

\section{Acknowledgments}

This work was supported by the scientific research project of Shaanxi Provincial Department of Education (18jk1067) and the scientific research project of Xi'an Eurasia University (2019xjzk03).

\section{References}

1. P. Wang, L.T Li, Y.Z. Ding, Building Structure, 49 (S2) (2019)

2. P.N. Hiremath, S.C. Yaragal, Constr. Build. Mater. 141 (2017)

3. P. Richard, M.H. Cheyrezy, ACI Spring Convention, San Francisco, SP.144(24), (1994)

4. P. Richard, M. Cheyrezy, Cem. Concr. Res. 25 (1995)

5. Y. Wang, M.Z. An, Z.R. Yu, et al. Constr. Build. Mater. 172 (2018)

6. S.J. Cao, X.M. Hou, Q. Rong, Cement. Concrete Comp. 110 (2020)

7. K. Wang, J. Lin, W.D. Yang, Concrete 11 (2017)

8. L.F. Yang, Q. Ma, B. Yu, Constr. Build. Mater. 161 (2018)

9. R.D. Jia, S.J. Fu, New Building Materials 3 (2019)

10. X.E. Yan, China Building Materials Science and Technology 2 (2017)

11. J.B. Wang, D.T. Niu, Chinese Journal of Civil Engineering 51(2)(2018)

12. Y.F. Ruan, B.G. Han, X. Yu, et al., Constr. Build. Mater. 162 (2018) 